\title{
VIRTUAL RESOLUTION ENHANCEMENT OF SCALE INVARIANT TEXTURED IMAGES USING STOCHASTIC PROCESSES
}

\author{
E. Konig, P. Chainais \\ LIMOS UMR 6158, University Blaise Pascal Clermont-Ferrand II - 63173 Aubière, France \\ \{emilie.koenig, pierre.chainais\}@isima.fr, http://www.isima.fr/ koenig, chainais
}

\begin{abstract}
We present a new method of magnification for textured images featuring scale invariance properties. The procedure preserves the visual aspect as well as the statistical properties of the initial image. An augmentation of information is performed by locally adding small scale details below the initial pixel size. This is made possible thanks to a family of scale invariant stochastic processes, namely compound Poisson cascades. This extrapolating procedure yields a potentially infinite number of magnified versions of an image. It allows for large magnification factors (virtually infinite) and is physically conservative: zooming out to the initial resolution yields the initial image back. This work is motivated by an application to images of the quiet Sun to quantitatively predict statistical and visual properties of images taken by a forthcoming high resolution telescope.
\end{abstract}

Index Terms - Image enhancement, resolution, image generation, scale invariance, stochastic processes, modeling.

\section{INTRODUCTION}

We present a new method to magnify random textured images by any factor (even much larger than 2) while preserving their visual aspect as well as their statistical properties. This procedure uses an augmentation of information by adding small scale details below the initial pixel size as the resolution gets (virtually) finer. We focus on random textured images such as those resulting from observations of the Sun by a spatial telescope or clouds by a satellite. Our approach uses a minimum of a priori on the original image. It relies on the fact that natural images and random textures usually present scale invariance properties $[1,2]$ to randomly extrapolate new details. Furthermore, we require that the content of the augmented image be consistent with the information available at larger scales from the original image. This consistency is defined by two requirements: the preservation of scale invariance properties of the initial image (statistical consistency) and a zoom out to the initial resolution must yield the original image back (physical consistency). One application domain is in solar physics where current observations are $1024 \times 1024$ images at resolution $1800 \mathrm{~km}$ (E.I.Telescope onboard SOHO)
[3]. Therefore, physicists are considering the design of a new spatial telescope with a much better resolution of $80 \mathrm{~km}$ which is about 25 times finer than that of EIT. However as the resolution gets finer, the flux of photons on the CCD sensor gets smaller so that the images may be under-exposed and unusable. Our approach proposes a way to simulate realistic images extrapolated from the current ones. The evolution of statistics at finer resolutions such as histograms could even be predicted. Another application lies in procedural texture synthesis for computer graphics. Our procedure could allow for an online enhancement of a texture depending on visualization conditions. More generally it could be used to enhance textured regions in a segmented image.

After some mathematical definitions we present our approach. Next, we illustrate the method on numerical examples and finally point to an application in solar physics.

\section{MATHEMATICAL PRELIMINARIES}

\subsection{Statistical scale invariance of images}

The statistics of natural images have been studied in depth [1] and have revealed two main properties: scale invariance and non-Gaussian statistics. This is also true for a large variety of "random textures" [2]. Note that some models for natural images can be used to synthesize random textures as well. The scale invariance of natural images is basically characterized by a power law Fourier spectrum $\propto 1 / k^{2-\eta}$ over a wide range of spatial frequencies. The notion of scale invariance can be defined for higher order statistics as well [4]. Let $T_{I}(r)$ be a multiscale transform (wavelets, box averages...) at scale $r$ of the image $I$ under study. The scale invariance property results in the power law scaling behavior $\mathbb{E} T_{I}(r)^{q} \propto r^{\zeta(q)}, q \in \mathbb{R}$ where $\mathbb{E}$ stands for mathematical expectation. The $\zeta(q)$ are called multiscaling exponents. They can be decomposed in a linear part $q H$ depending on a parameter $H$ and a non-linear part $\tau(q)$, such that $\tau(0)=\tau(1)=0$ and $H=\zeta(1)$. For instance in the case $\zeta(q)=q H$ (e.g. a fractional Brownian field), the image $I$ is said self-similar and its scale invariance is characterized by the parameter $H$ only. The $\tau(q)$ betray a multiscaling property: when $\tau(q) \neq 0$ a set of exponents is necessary to describe the scale-invariance of the image. It 
can be shown that the $\tau(q)$ depict the non-Gaussianity of the image statistics. For a multiscaling image, the parameters $H$ and $\tau(q)$ are linked to the power law Fourier spectrum which is $\propto 1 / k^{2+2 H+\tau(2)}$ [5]. Infinitely Divisible Cascades (IDC) are a family of multiscaling models for natural images recently proposed in [5]. Our approach uses a sub-family of IDC, namely Compound Poisson Cascades (CPC).

\subsection{Compound Poisson Cascades (CPC)}

We give below a brief presentation and refer to [5] for more details. This model is based on a multiplicative construction where smaller scales of an image inherit information from larger scales in a continuous way. Let $Q_{\ell}^{L}(\mathbf{x})$ be the pixel value at location $\mathbf{x}$; let $L$ and $\ell, L>\ell$, the limiting largest and smallest scales of the resulting image. Let $\left(\mathbf{x}_{i}, r_{i}\right)$ a Poisson point process with: (i) $\mathbf{x}_{i}$ uniformly distributed in the $2 \mathrm{D}$ plane, ensuring the homogeneity of $Q_{\ell}^{L}$ in space; (ii) $\ell \leq$ $r_{i} \leq L$ distributed with density $1 / r^{3}$ (so that the final texture is mathematically well defined if $\ell>0$ only). The $\left(\mathbf{x}_{i}, r_{i}\right)$ are marked by i.i.d. positive random variables $W_{i}$ called multipliers, independent of the $\left(\mathbf{x}_{i}, r_{i}\right)$. Let $f(\mathbf{x})$ be a non negative function with compact support. For all $\mathbf{x}$ of the image, the CPC $Q_{\ell}^{L}(\mathbf{x})$ is defined by

$$
Q_{\ell}^{L}(\mathbf{x})=C \prod_{i} W_{i}^{f\left(\frac{\mathbf{x}-\mathbf{x}_{i}}{r_{i}}\right)}
$$

where $C$ is a normalization coefficient such that $\mathbb{E} Q_{\ell}^{L}=1$. Such models are homogeneous but very irregular objects and correspond from a mathematical standpoint to distributions with log compound Poisson law. Their scale invariance is characterized by $H=0$ and $\tau(q)=q\left(\mathbb{E} W_{i}^{q}-1\right)+1-\mathbb{E} W_{i}^{q}$ for scales between $\ell$ and $L$. Their Fourier spectrum obeys a power law $\propto 1 / k^{2+\tau(2)} . Q_{\ell}^{L}(\mathbf{x})$ is equal in distribution $(\doteq)$ to $Q_{\ell / L}^{1}(\mathbf{x} / L)$. Its variance is given by:

$$
\sigma_{Q_{\ell}^{L}}^{2}=(\ell / L)^{\tau(2)}-1
$$

An essential property of CPC is that for any $0<r_{2} \leq r_{1} \leq$ $r_{0}, Q_{r_{2}}^{r_{0}}$ obeys a multiplicative multiscaling decomposition

$$
Q_{r_{2}}^{r_{0}}=Q_{r_{1}}^{r_{0}} \cdot Q_{r_{2}}^{r_{1}}
$$

where $Q_{r_{1}}^{r_{0}}$ and $Q_{r_{2}}^{r_{1}}$ are two independent CPC with the same $\tau(q)$. One can create a larger family of images by using fractional pseudo-integration and derivation. The fractional pseudo-integration, denoted by the operator $\mathcal{I}_{H}$, is performed in practice by a $1 /\|k\|^{H}$ low-pass filter in the Fourier domain for $\|k\|>0$ (the singularity at $k=0$ is treated separately). The fractional pseudo-derivation, denoted by $\mathcal{D}_{H}=\mathcal{I}_{H}^{-1}$, is the inverse of the integration and is performed in practice by a $\|k\|^{H}$ high-pass filter. Note that these two operations preserve the scale invariance of the original image but modify the scaling exponents $\zeta(q)$ by adding, respectively subtracting, a linear part $q H$. Thus, CPC may be used to model very

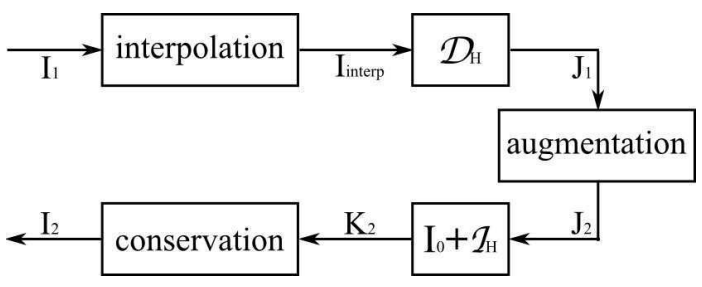

Fig. 1. Schematic view of the magnification process.

rough images (or textures) with $\zeta(1)=0$ as well as smoother images with $\zeta(1)=H>0$.

\section{VIRTUAL RESOLUTION ENHANCEMENT}

\subsection{The approach}

Our purpose is to (virtually) refine the resolution of scale invariant textured images thanks to an adapted augmentation of information. We require the augmentation to be consistent with the initial image, which means that (i) the resulting image has the same scale invariance properties as the original one and (ii) zooming out the magnified image to the initial resolution yields the initial image back. To this aim, one must add details with well suited properties at scales below the pixel size of the original image. These details can be generated from the extrapolation of the initial image wavelet coefficients assuming a local power law behavior as in [6]. However, the new wavelet coefficients strongly depend on the wavelet used for the image decomposition and on the estimation of the local regularity. Another possibility is to use I.F.S. (Iterated Function Systems) [7]. Interpolation using I.F.S. consists in applying another iteration of the initial I.F.S. determined thanks to the collage theorem [7]. These methods preserve scale invariance but they are deterministic and do not extrapolate the multifractal behavior of the initial image. Our method is more flexible since it adds scale invariant and multifractal information at high resolution in a stochastic manner. A fundamental assumption which is also a limitation is that the scale invariance properties of the original image are known, which is the case for, e.g., synthetic textures, or images that have been previously analyzed. The main thrust is to a priori describe the initial image by a global model using a fractionally pseudo-integrated CPC. This model is used to derive a local procedure to add random correlated details in every pixel of the initial image. Eventually, a renormalization step ensures that the procedure is conservative in the sense that zooming out to the initial resolution gives the original image back.

\subsection{Magnification procedure}

The initial image is supposed to have some random texture looking, e.g. taken from a turbulent physical system such as 
the quiet Sun characterized by the scale invariance parameters $H$ and $\tau(q)$ estimated from a prior multifractal analysis [3]. The image is a priori modeled by

$$
I_{1}=I_{0}+\alpha \mathcal{I}_{H}\left\{Q_{r_{1}}^{r_{0}}-\left\langle Q_{r_{1}}^{r_{0}}\right\rangle\right\}
$$

where \langle\rangle stands for spatial averaging and $I_{0}$ for the average intensity of the image; the fluctuation term is a fractionally pseudo-integrated CPC with zero mean, amplified by a factor $\alpha$. The scales $r_{0}$ and $r_{1}$ stand for the largest scale and the finest scale (the pixel size) of the image. We stress that this global model is not aimed at describing the complete image but is only used to derive a local procedure of augmentation. The main idea of this magnification procedure is to replace $Q_{r_{1}}^{r_{0}}$ by $Q_{r_{2}}^{r_{0}}$ in (4) by using the multiscale decomposition of CPC (3) to get an image $I_{2}$ at resolution $r_{2} \leq r_{1}$. The procedure is schematically represented in fig. 1 .

The first step magnifies $I_{1}$ by resampling it at $r_{2} \leq r_{1}$ using a spline interpolation [8] leading to a smoothed version $I_{\text {interp }}$ of $I_{1}$. A fractional derivation yields $J_{1}=\mathcal{D}_{H} I_{\text {interp }}$ which corresponds to the term $\alpha\left(Q_{r_{1}}^{r_{0}}-\left\langle Q_{r_{1}}^{r_{0}}\right\rangle\right)$ in (4). By using the multiscale decomposition (3) in this model of $J_{1}$, we define a new image $J_{2}$ at resolution $r_{2} \leq r_{1}$ :

$$
J_{2}=J_{1} \cdot Q_{r_{2}}^{r_{1}}+\alpha\left\langle Q_{r_{1}}^{r_{0}}\right\rangle\left(Q_{r_{2}}^{r_{1}}-\left\langle Q_{r_{2}}^{r_{1}}\right\rangle\right)
$$

where $Q_{r_{2}}^{r_{1}}$ contains the new information at scales smaller than $r_{1} . Q_{r_{2}}^{r_{1}}$ is independent of $Q_{r_{1}}^{r_{0}}$; it is generated using (1) with the same parameters as $Q_{r_{1}}^{r_{0}}$. Note that since $Q_{r_{2}}^{r_{1}}(\mathbf{x}) \doteq$ $Q_{r_{2} / r_{1}}^{1}\left(\mathbf{x} / r_{1}\right)$, the information brought by the CPC between scales $r_{1}$ and $r_{2}$ depends on the magnification factor $r_{2} / r_{1}$ only. The first term in (5) mainly describes the augmentation of information in $J_{1}$ at scales $r_{2} \leq r \leq r_{1}$. It ensures the coupling between the initial image $J_{1}$ and the new details $Q_{r_{2}}^{r_{1}}$. The second term ensures that the energy level of the new details is consistent with the initial image. $J_{2}$ is then an image with the same $\tau(q)$ as $I_{1}$ and a power law Fourier spectrum over a larger range of spatial frequencies. Note that each realization of $Q_{r_{2}}^{r_{1}}$ will produce one possible realization of the magnification of the original image among a potential infinity. The parameter $\alpha$ depends on $r_{1} / r_{0}$ and can be estimated using (2):

$$
\widehat{\alpha}=\sqrt{\frac{\sigma_{J_{1}}^{2}}{\left(\widehat{r_{1} / r_{0}}\right)^{\tau(2)}-1}}
$$

where $\tau(2)$ can be estimated from the power law Fourier spectrum $\propto 1 / k^{2+2 H+\tau(2)}$ or from some multiscaling analysis. As $\mathbb{E}\left\langle Q_{r_{1}}^{r_{0}}\right\rangle=1$, we set $\left\langle Q_{r_{1}}^{r_{0}}\right\rangle=1$ in (5). Section 4.2 details the sensitivity of our approach to parameters. The pseudointegration step yields $K_{2}=I_{0}+\mathcal{I}_{H} J_{2}$. Finally, the magnified image $I_{2}$ results from a renormalization of $K_{2}$ which makes the procedure "conservative". While (5) adds information at scales $r_{2} \leq r \leq r_{1}$, it slightly affects scales larger than $r_{1}$ as well. Therefore, we impose that the sum of intensities in regions of $I_{2}$ corresponding to a pixel of size $r_{1}$ in $I_{1}$ equals

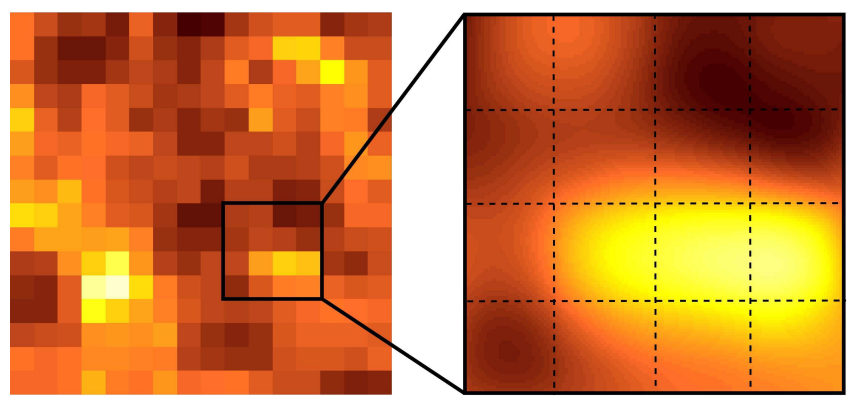

$\Downarrow \quad$ (a) $\Uparrow$

$\Downarrow \quad$ (b) $\Uparrow$

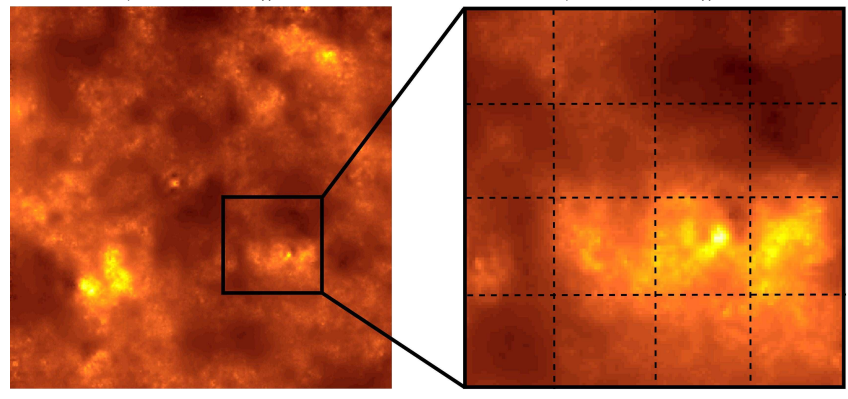

(c)

(d)

Fig. 2. Illustration of our magnification procedure: (a) initial $16 \times 16$ image, (b) zoom in the ' $\times 32$ ' interpolated image contained in the $4 \times 4$ black square of the initial image, (c) ' $\times 32$ ' magnified image with augmentation, (d) zoom in the black square region. The dotted lines correspond to initial pixels.

the pixel value in $I_{1}$. The augmentation procedure and the conservation step are non-linear and local operations so that a direct ' $\times 32$ ' magnification is very different from five successive ' $\times 2$ ' magnifications. Ideally, the ratio $r_{2} / r_{1}$ should be close to 1 to preserve the scale invariance of the original image and limit any "boxing" artifact due to the conservation step. In the present work, and for obvious practical reasons, we use $r_{2} / r_{1}=1 / 2$.

\section{COMPUTATIONAL ILLUSTRATION}

\subsection{Main properties}

We illustrate our approach in the ideal case where the initial image is precisely of the form given by (4). A set of initial images of size $32 \times 32$ is built with $\alpha=8$ and $I_{0}=23$, for $r_{0}=1$ and $r_{1}=1 / 32$ and with $H=0.7$. In these examples we have chosen $\tau(q)=-\left((1+0.7)^{q} /(1+q 0.7)-1\right)$ which leads to $\tau(2)=-0.20$. A magnification ' $\times 32$ ' is performed as five ' $\times 2$ ' elementary magnifications leading to $1024 \times 1024$ images. Fig. 2 compares typical results obtained by simple interpolation and by our magnification procedure. The same procedure can be applied to images of the Sun with the same visual and quantitative results (see our webpages).

Here, we assume that $\alpha, r_{1} / r_{0}$ and $\tau(2)$ are exactly 


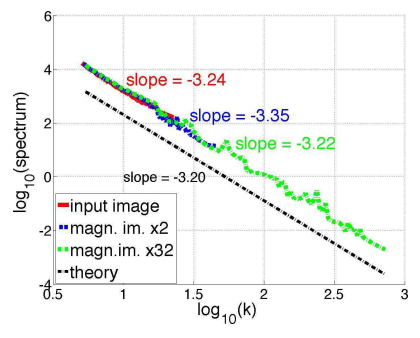

(a)

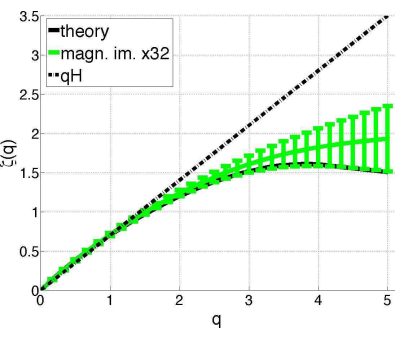

(b)
Fig. 3. (a) Power law Fourier spectrums and (b) multifractal exponents of ' $\times 2$ ' and ' $\times 32$ ' magnified images.

known. Fig. 2 clearly illustrates the augmentation of information by the introduction of new small scale details inside initial pixels while a simple interpolation only smoothes the image. Moreover, reducing (by aggregation) the resolution of $I_{2}$ back to $r_{1}$ yields $I_{1}$ back. Fig. 3(a) presents a log-log plot of the Fourier spectra of $I_{1}$ and $I_{2}$ after magnifications by factors 2 and 32 . We see that the power law Fourier spectrum of $I_{1}$ has been preserved and extended to higher frequencies without any discontinuity. Fig. 3(b) shows that the multiscaling exponents estimated from $I_{2}$ are close to the theoretical ones within reasonable error bars. Thus we have shown, at least on these examples, that our magnification procedure adds new information to the original image while (i) enhancing its visual aspect, (ii) preserving its scale invariance properties and (iii) being conservative with respect to zoom in and out operations. These tests have been applied to a wide variety of multifractal images and have given similar results.

\subsection{Sensitivity to parameters}

We now consider the realistic situation where $\alpha$ and the ratio $r_{1} / r_{0}$ are unknown. We still assume that the scaling exponents of $I_{1}$ are known from a prior analysis. As seen in (6), $\alpha$ depends on $r_{1} / r_{0}$ so that an error on $\widehat{r_{1} / r_{0}}$ may lead to an error on $\widehat{\alpha}$. The scale $r_{1}$ is the smallest scale available, the pixel size. Therefore, the estimation of $\alpha$ is directly linked to the estimation of the larger scale $r_{0}$ in the image, which may be in general smaller but sometimes larger than the image size. On one hand, if $r_{0}$ is over-estimated, the power of details is too small, the final image misses some energy at high frequencies. On the other hand, if $r_{0}$ is under-estimated, the power of details is too big and results in too much energy at high frequencies. Fortunately, a precise estimation of $r_{0}$ is not so crucial for our procedure to be efficient. In practical cases, the maximum error on $r_{0}$ will be within a factor 2 which leads to an error on $\alpha$ of $10 \%$. Furthermore, the fractional pseudointegration as well as the conservation step tends to reduce the uncertainty on $\widehat{\alpha}$. In the end, the error is close to negligible. As a conclusion, the accuracy of the estimation of $r_{1} / r_{0}$ and $\alpha$ is not critical.

\section{CONCLUSION}

We have presented a new approach to the magnification of random textured images featuring scale invariance properties by using an augmentation of information. Our method locally adds small scale details below the pixel size using stochastic processes. It preserves the original scale invariance properties, extends them to smaller scales (statistical consistency) and is conservative (physical consistency), that is a zoom out of the magnified image yields the original image back. One originality of our approach lies in the synthesis of new details in a random manner so that this magnification/extrapolation can potentially produce infinitely many magnified versions of an image. An application to the detailed modeling of quiet Sun images which have revealed multiscaling properties [3] is now the subject of ongoing work. Our approach opens new insights to a quantitative prediction of visual quality and statistics of future observations at a finer resolution (1 pixel $=80 \mathrm{~km})$ given present low resolution observations (1 pixel $=1800 \mathrm{~km}$ ). Another application under study is in image processing for the enhancement of textured regions. Other illustrations of this magnification method are available at www.isima.fr/ $\sim$ koenig and $\sim$ chainais.

\section{REFERENCES}

[1] A. Srivastava, A.B. Lee, E.P. Simoncelli, and S-C. Zhu, "On advances in statistical modeling of natural images," Jour. of Math. Im. and Vision, vol. 18, pp. 17-33, 2003.

[2] D.S. Ebert, F.K. Musgrave, D. Peachey, K. Perlin, and S. Worley, Texturing and Modeling: a procedural approach, Morgan Kaufmann Publishers Inc., 2002.

[3] V. Delouille, P. Chainais, and J.-F. Hochedez, "Quantifying and containing the curse of high resolution coronal imaging," Annales Geophysicae, vol. 26, no. 10, pp. 3169-3184, 2008.

[4] A. Turiel, G. Mato, N. Parga, and J-P. Nadal, "Selfsimilarity properties of natural images resemble those of turbulent flows," Phys. Rev. Lett., vol. 80, no. 5, pp. 1098$1101,1998$.

[5] P. Chainais, "Infinitely divisible cascades to model the statistics of natural images," IEEE Trans. on P.A.M.I., vol. 29, no. 12, pp. 2105-2119, 2007.

[6] P. Legrand and J. Levy-Vehel, "Hölderian regularitybased image interpolation," in IEEE ICASSP, 2006.

[7] M.F. Barnsley, Fractals Everywhere, Academic Press, 1988.

[8] M. Unser, A. Aldroubi, and M. Eden, "Enlargement or reduction of digital images with minimum loss of information," IEEE Trans. on Im. Proc., vol. 4, no. 3, pp. 247-258, 1995. 